\title{
EARTH ROTATION FROM RADIO INTERFEROMETRIC TRACKING OF GPS SATELLITES
}

\author{
R. I. Abbot, R. W. King, Y. Bock, C. C. Counselman III \\ Department of Earth, Atmospheric, and Planetary Sciences \\ Massachusetts Institute of Technology \\ Cambridge, MA 02139
}

\begin{abstract}
Radio-interferometric tracking of the Global Positioning System (GPS) satellites offers a new technique for regular monitoring of variations in the earth's rotation. The observations are sensitive to pole position and length-of-day, at a level of precision which may make this technique competitive with satellite and lunar laser ranging and very long baseline interferometry (VLBI). The present limitations are the number of satellites and tracking stations available and inadequate modeling of nongravitational forces on the satellites. The potential advantages are rapid turn-around and minimal incremental cost. We have performed a preliminary analysis using six days of observations from a four-station network. Comparison of earth rotation values from our GPS analysis with values obtained by VLBI and laser ranging reveals differences after five days of $0.9 \mathrm{~ms}$ in UT1, 0.04" in $\mathrm{x}$ and $0.07^{\prime \prime}$ in $\mathrm{y}$. These differences reflect errors in the GPS determinations due primarily to inadequate modeling of nongravitational forces.
\end{abstract}

\section{INTRODUCTION}

Like the Navy Navigation (TRANSIT) satellites and LAGEOS, the satellites of the Global Positioning System (GPS) provide an opportunity for monitoring the earth's rotation as a by-product of routine orbit determination. The high altitude $(20,000 \mathrm{~km})$ of the GPS satellites makes their orbits less sensitive than those of the other satellites to errors in the models for the earth's gravity field and the solid-earth and ocean tides. However, the GPS satellites are more affected by non-gravitational forces, arising from direct and reflected radiation pressure on their large solar panels, from accidental discharge of battery and thruster gases, and from unbalanced forces produced by the attitude control system. The best accuracy in GPS orbit determination, achieved with three or four tracking stations and arc lengths of a few days, has been of the order of 1 part in $10^{7}$, equivalent to an error of $0.02^{\prime \prime}$ in the orientation of the earth. However, future increases in the numbers of tracking stations and satellites, together with improved modeling of the orbital dynamics, promise a significant reduction in the errors in earth rotation from GPS tracking. The accumulation of errors due to non-gravitational forces will necessarily limit the contribution of GPS to the determination of shortterm variations in the rotation. Nevertheless, the low cost of data acquisition and processing make GPS an attractive component of a service for rapid determination and distribution of earth rotation parameters. 
There are secondary benefits to estimating earth-rotation parameters as part of GPS orbital analyses. The operational orbital adjustment performed by the Defense Department and civilian agencies can be freed from dependence on an external source of rotation information, which might not be available in a timely fashion. For high-precision geodetic applications, comparison of the values of earth-rotation parameters determined from GPS with values determined from very long baseline interferometry (VLBI) and satellite and lunar laser ranging observations provides an independent check on the accuracy of the GPS orbits.

In order to better understand the use of GPS for determining variations in the earth's rotation, and to ascertain the current accuracy, we performed a limited, preliminary analysis using observations performed over a 6-day period in March 1985. In this paper we describe the method used in our analysis and compare the earth-rotation parameters estimated with results from VLBI and satellite laser ranging (SLR).

\section{METHODS OF ANALYSIS}

We used observations of carrier phase obtained from four stations during the GPS High Precision Baseline Test of March-April 1985 [Melbourne et al., 1985]. Observations were obtained by three Air Force Geophysics Laboratory receivers which we had installed at the Haystack Observatory in Westford, Massachusetts, at the U. S. Naval Observatory Time Service Alternate Station in Richmond, Florida, and at the G. R. Agassiz Station of the Harvard College Observatory in Ft. Davis, Texas. We also used observations from a Texas Instruments (TI) 4100 receiver operated by TI personnel at the Owens Valley Radio Observatory in Big Pine, California. We combined dual-frequency phase observations from the four stations, for five GPS satellites, over six consecutive days, using the double-differencing algorithm described by Bock et al. [1986].

In order to construct an accurate terrestrial reference system for our analysis, we compared coordinates determined by VLBI and by SLR for four sites which are geographically well-distributed and whose positions have been well determined by both VLBI and SLR. The SLR coordinates were from the "84L3" analysis performed at the University of Texas Center for Space Research (CSR) (R. Eanes, private communication, 1985; see also Tapley et al.[1985]). The VLBI coordinates were from the "Series 85 Oct 26" analysis of the National Geodetic Survey [IRIS, 1985]. We used results of local surveys to relate the SLR and VLBI coordinates, and determined a 7-parameter transformation between the two systems. We applied the transformation parameters to the VLBI-determined coordinates of the Westford, Ft. Davis, Richmond, and Big Pine antennas to obtain coordinates of these sites in the (CSR 84L3) SLR system. Finally, we determined the coordinates of the GPS antenna at each site from local surveys.

We computed the orbits of the GPS satellites by direct numerical integration of the equations of motion in inertial coordinates. Our force model included luni-solar perturbations from numerically integrated models and earth-gravitational perturbations from the GEM L2 gravity field [Lerch et al., 1982] truncated to $8 \times 8$. The effect on the satellites of direct solar radiation pressure was accounted for using an isotropic (flatplate) model with one free parameter for each satellite. We also included, with a free parameter, a force along the $y$-axis of the spacecraft. This "y-bias" force was suggested several years ago by W. Feess of Aerospace Corporation to account for bending of the solar panels and for unbalanced thermal radiation and absorption at the base of the panels [Fliegel et al., 1985]. In several years of orbital analyses by the Defense 
Department, the y-bias model has been found more effective than other ad hoc force models (e.g., an along-track or "drag" force model) in improving the predictive quality of the estimated orbits (P. E. Beveridge and J. T. Carr, Naval Surface Weapons Center, private communications, 1984).

We analyzed the observations from the 6-day arc simultaneously, estimating six initial conditions and the two non-gravitational parameters for each satellite, and Universal Time (UT1) and pole-position parameters for five of the six days. Since we have no tie through the GPS satellites to the right-ascension origin of the conventional celestial (nearly inertial) reference system (CCRS), we fixed UT1 on the first day at a value determined from a combination of VLBI and SLR results [King et al., 1984]. Since the GPS observations were much less sensitive to the average position of the earth's pole than to polar motion during the observation span, we also fixed the $x$ and $y$ coordinates of the pole on the first day to the VLBI-SLR-determined values.

\section{RESULTS}

Table I shows the differences after five days between the UT1, $x$, and $y$ values determined from the GPS observations, and the values computed from VLBI and SLR. The smallest difference is in UT1, and the largest in the y-coordinate of the pole. Since the uncertainties in the VLBI/SLR values are less than $0.1 \mathrm{~ms}$ in UT1 and 0.002" in pole position, the differences in Table I reflect mainly the effect of errors our models of the GPS orbits.

TABLE I. Differences after 5 days between GPS and

VLBI/SLR determinations of earth rotation parameters.

\begin{tabular}{lll}
\hline Parameter & Difference & Difference in radians \\
\hline UT1 & $0.9 \mathrm{~ms}$ & $6 \times 10^{-8}$ \\
x & $0.04^{\prime \prime}$ & $2 \times 10^{-7}$ \\
y & $0.07^{\prime \prime}$ & $3 \times 10^{-7}$ \\
\hline
\end{tabular}

The largest source of orbit error is almost certainly non-gravitational force on the satellites. Such forces can act in any direction with respect to the satellite's motion, but the along-track component alters the orbital period and therefore dominates after a day ( 2 revolutions) or more. Since most of the satellites cross North America in a northsouth direction, the effect of an along-track orbital position error can resemble that of an error in the latitudes of the tracking stations. For North American stations, variation of latitude corresponds to a change in the y-coordinate of the pole.

To test the hypothesis of unmodeled non-gravitational forces, we divided the observations into two 3-day spans. For each span we estimated the six initial conditions of the satellites and the solar radiation pressure and $y$-bias parameters. The changes between the two spans in the values of the non-gravitational force parameters 
were of the order of $2 \times 10^{-10} \mathrm{~m} / \mathrm{s}^{2}$. Over six days, an acceleration of this magnitude would produce an error in the along-track component of the orbit of 2-15 $\mathrm{m}$, depending on the orientation of the acceleration with respect to the satellite's orbit [Rizos and Stolz, 1985]. An error of $15 \mathrm{~m}$ in orbital position is equivalent to an error of $0.1^{\prime \prime}$ in the earth's orientation.

Acknowledgement This research was sponsored by Contract F19628-86-K-0009 with the Air Force Geophysics Laboratory.

\section{REFERENCES}

Abbot, R. I., Y. Bock, C. C. Counselman III, R. W. King, S. A. Gourevitch, and B. J. Rosen, Interferometric determination of GPS orbits, in Proc. 1st Inter. Sym. on Precise Positioning with GPS, C. Goad, edit., NOAA, Rockville, Md, pp. 63-72, 1985.

Bock, Y., S. A. Gourevitch, R. I. Abbot, C. C. Counselman III, R. W. King, and R. I. Abbot, Interferometric analysis of GPS phase observations, Man. Geod., 11, No. 4, 1986.

Fliegel, H. F., W. A. Feess, W. C. Layton, and N. W. Rhodus, The GPS radiation force model, Proc. 1st Inter. Sym. on Precise Positioning with GPS, C. Goad, edit., NOAA, Rockville, Md, pp. 113-120, 1985.

IRIS Earth Orientation Bulletin, No. 21, issued by Subcommission International Radio Interferometric Surveying, National Geodetic Survey, NOAA, Rockville, Md, 1985.

King, R.W., B. A. Kolaczek, and I. I. Shapiro, Accuracies of recent observations of the Earth's rotation (abstract), Eos Trans. AGU, 65, 187, 1984.

Lerch, F. S., S. M. Klosko, and G. B. Patel, A refined gravity model for LAGEOS (GEM L-2), Geophys. Res. Lett., 9, 1263-1266, 1982.

Melbourne, W., R. Anderle, M. Feissel, R. King, D. McCarthy, D. Smith, B. Tapley, and R. Vicente, Project MERIT Standards, Circ. No. 167, U. S. Naval Observatory, Washington, D.C., 1983.

Melbourne, W. G., J. R. Clynch, B. H. Hager, R. L. Merrell, S. Moseley, B. E. Schutz, and R. R. Ware, The March 1985 demonstration of GPS-based geodesy: an overview (abstract), Eos Trans. AGU, 66, 843, 1985.

Rizos, C., and A. Stolz, Force modelling for GPS satellite orbits, Proc. 1st Inter. Sym. on Precise Positioning with GPS, C. Goad, edit., NOAA, Rockville, MD, 87-98, 1985.

Tapley, B. D., B. E. Schutz, and R. J. Eanes, Station coordinates, baselines, and earth rotation from LAGEOS laser ranging: 1976-1984, J. Geophys. Res., 90, 92359248, 1985. 


\section{DISCUSSION}

Herring: Was the growth of the difference between GPS EOP and the combined solution systematic or random? What was the RMS scatter about the systematic slope?

Reply by Abbot: There was a systematic slope in the differences. From the table which was shown, UT1 built up from 0.3 milliseconds after one day up to 0.9 milliseconds after five days with an rms of 0.09 milliseconds. For $\mathbf{x}$ of the pole, the buildup went from 0.005 arcseconds after one day up to 0.04 arcseconds after five days with an rms of 0.001 arcseconds; and similarly for $y$ of the pole, from 0.03 arcseconds to 0.07 arcseconds after five days with an rms of 0.003 arcseconds.

Eubanks: I was a little surprised by your results, which seemed to show that the polar motion error grew 3-5 times as fast as the UT1 error, contrary to the Lageos experience. Is this true, and if so why?

Reply by Abbot: We seem to do very well with the orbital node, and thus the UT1. We always do better in crose track than along track, and, since we currently only observe the spacecraft as it passes over North America, the large along track error translates into a large polar motion error.

Dickey: The GPS is not a mature system as yet, given both the number of satellites in orbit and the tracking system. Do you have estimates of improved accuracies given more satellites and a more complete tracking system?

Reply by Abbot: No, we haven't done any quantitative studies to investigate what the improved accuracies would be if additional satellites and tracking stations could be used. 